\title{
Excitation of oblique whistler waves in magnetosphere and in interplanetary space at 1 A.U.
}

\author{
R. S. Pandey and K. D. Misra \\ Department of Applied Physics, Institute of Technology Banaras Hindu University, Varanasi-221005, India
}

(Received May 9, 2001; Revised October 5, 2001; Accepted October 23, 2001)

\begin{abstract}
The oblique whistler waves have been studied having $\mathbf{k}$ vector at an angle to magnetic field for a generalized distribution function reducible to bi-maxwellian and loss-cone. The dispersion relation and growth rate have been obtained for oblique whistler mode instability incorporating the trajectory of the particles, in the presence of perpendicular a-c electric field by method of characteristic solutions. The effects of distribution function and beam effect have been discussed for the space plasma at magnetospheric height and at 1 A.U. The results are compared with satellite observations and reported results obtained by other techniques. Excitation of two separate, but simultaneous left hand polarized whistler mode at 1 A.U. by electron been are demonstrated.
\end{abstract}

\section{Introduction}

Plasma waves in the vicinity of the magnetopause at ELF/VLF frequencies propagating waves have been studied by many researchers (Rycroft, 1972; Misra and Singh, 1977, 1980; Misra et al., 1979; LaBelle and Treuman, 1988; Sazhin, 1988, 1993; Tsurutani, 1981, 1989; Misra and Haile, 1993; Misra and Pandey, 1995). Intensification of these waves are general features of magnetopause crossings. Flux transfer events were particularly pronounce at frequencies below electron gyrofrequency range consistent with ISEE-2 and 3 and AMPTE-UKS plasma wave data. AMPTE-UKS satellite recorded on board and measured wave normal angles of whistler mode waves. Similar study was also done by Kimura and Matsuo (1982). Whistler mode instabilities were analyzed using data from magnetosphere and electron experiment on board ISEE and AMPTE-UKS (Korth et al., 1984; Ward et al., 1985; Kennel et al., 1986). The distribution function was seen like having mixture of two bi-Maxwellian of density of $10^{6} / \mathrm{m}^{3}$, with anisotropy of nearly 2 and energies ranging from 12 ev to 2.5 $\mathrm{keV}$ (Shah et al., 1985). The growth of whistler wave was also investigated from an anisotropic electron beam of various electrostatic and electromagnetic wave modes at various propagation angle using a series of one-dimensional simulation (Zhang et al., 1993). However wave propagation was still restricted to a single fixed direction relative to the magnetic field. Devine et al. (1995) generalized oblique whistler mode instability in one and two-dimensional simulations. Gary (1992) and Gary et al. (1993) studied whistler mode instabilities in the magnetosheath in electron-proton plasma with temperature anisotropy. Gary and Madland (1985) in their study of whistler instability observed that maximum growth rate are very sensitive to background plasma.

Copy right (C) The Society of Geomagnetism and Earth, Planetary and Space Sciences (SGEPSS); The Seismological Society of Japan; The Volcanological Society of Japan; The Geodetic Society of Japan; The Japanese Society for Planetary Sciences.
Electron beam excitation of upstream waves in the whistler mode frequency range was also studied in interplanetary space at 1 A.U. Both parallel and obliquely propagating solutions were considered (Wong and Smith, 1994). Whistler waves have been observed in upstream from obliquely propagating shock waves in both simulations and space plasma (Lyn and Kan, 1990; Pantellini et al., 1992 and references cited there in). For a solar wind type plasma expected wavelength are of the order of inertial length and depend on obliquely propagating speed and field orientation (Thomas et al., 1990; Winske et al., 1990). High frequency waves have been observed by ISEE-3 in the distant upstream plasma (Kennel et al., 1980, 1986) resulting from solar wind rather than from shock origin in accordance with Gary and Feldman (1997)

Recently competing processes of oblique whistler mode instabilities were studied due to anisotropic electron beam having linear results as well as two-dimensional particle simulations (Borda de Agua et al., 1996). This has a direct relevance for interpretation of wave activities observed in Earth's magnetosphere by the GEOS1, GEOS2 and GEOTAIL satellites or in uranian bow shock by Voyger2 were proposed.

In the recent past electric field measurements on satellites, such as S3-3 and Viking have given fluctuations the frequency range $0.1-100 \mathrm{~Hz}$ and higher ranging up to few $\mathrm{KHz}$ (Block and Falthammer, 1990 and reference cited there in). Parallel and perpendicular a-c electric field measurements were also reported in Earth's bow shock regions (Mozer et al., 1978; Wygant et al., 1987; Lindqvist and Mozer, 1990). Recently particle and fields measurements were also reported by DMSPF8 and CRRES satellites (Maynard et al., 2000). Therefore, it attaches significance to see how for, whistler emissions are affected if such a field is simultaneously present. Such small magnitude fields may also be produced due to many other types of fluctuations.

In the present paper therefore, oblique whistler mode in- 
stabilities were performed having [ $\mathbf{k}_{\perp}$ and $\mathbf{k}_{\|}$] wave numbers for a generalized drifting distribution function, reducible to bi-maxwellian and loss-cone distribution, in the presence of perpendicular a-c electric field by method of characteristics solutions. In the section second, using the details of particle trajectories (Misra and Pandey, 1995) dispersion relation have been given. Analytical expressions for growth rate have been evaluated. In the section third, results have been discussed and applied to magnetospheric as well as in interplanetary space plasma at 1 A.U. lastly conclusion are outlined.

\section{Dispersion Relation and Growth Rate}

Using the method and technique of Misra and Pandey (1995) the unperturbed trajectories of charged particles including a-c electric field and equilibrium distribution function $f_{0}(\mathbf{v})$ are written as:

$$
\begin{aligned}
X_{0}= & X+\left(\mathbf{v}_{y} / \omega_{c s}\right)+\left(1 / \omega_{c s}\right)\left[\mathbf{v}_{x} \sin \omega_{c s} t^{\prime}-\mathbf{v}_{y} \cos \omega_{c s} t^{\prime}\right] \\
& +\left(\Gamma_{x} / \omega_{c s}\right)\left[\left(\omega_{c s} \sin v t^{\prime}-v \sin \omega_{c s} t^{\prime}\right) /\left(\omega_{c s}^{2}-v^{2}\right)\right] \\
Y_{0}= & Y+\left(\mathbf{v}_{x} / \omega_{c s}\right)\left(1 / \omega_{c s}\right)\left[\mathbf{v}_{x} \cos \omega_{c s} t^{\prime}-\mathbf{v}_{y} \sin \omega_{c s} t^{\prime}\right] \\
& -\left(\Gamma_{x} / v \omega_{c s}\right) \\
& \cdot\left[1+\left\{\left(v^{2} \cos \omega_{c s} t^{\prime}-\omega_{c s}^{2} \cos v t^{\prime}\right) /\left(\omega_{c s}^{2}-v^{2}\right)\right\}\right] \\
Z_{0}= & Z-\mathbf{v}_{z} t^{\prime}
\end{aligned}
$$

and the velocities are

$$
\begin{aligned}
\mathbf{v}_{x 0}= & \mathbf{v}_{x} \cos \omega_{c s} t^{\prime}-\mathbf{v}_{y} \sin \omega_{c s} t^{\prime} \\
& +\left\{v \Gamma_{x} /\left(\omega_{c s}^{2}-v^{2}\right)\right\}\left(\cos v t^{\prime}-\cos \omega_{c s} t^{\prime}\right) \\
\mathbf{v}_{y 0}= & \mathbf{v}_{x} \sin \omega_{c s} t^{\prime}+\mathbf{v}_{y} \cos \omega_{c s} t^{\prime} \\
& -\left\{\Gamma_{x} /\left(\omega_{c s}^{2}-v^{2}\right)\right\}\left(\omega_{c s} \sin v t^{\prime}-v \sin \omega_{c s} t^{\prime}\right) \\
\mathbf{v}_{z 0}= & \mathbf{v}_{z} .
\end{aligned}
$$

Where, $\omega_{c s}=\left(e_{s} \mathbf{B}_{0}\right) / m_{s}$ is the cyclotron frequency of species $s$ and $\Gamma_{x}=\left(e_{s} \mathbf{E}_{0 x} / m_{s}\right)$ and a-c electric field is varying as $\mathbf{E}=\mathbf{E}_{0 x} \sin v t, v$ being the angular a-c frequency.

$$
\begin{aligned}
f_{0}(\mathbf{v})= & \left\lfloor\left(n_{0} \mathbf{v}^{2 j}\right) /(\pi)^{3 / 2} \alpha^{2(j+1)} \alpha_{\|} j !\right\rfloor \\
& \cdot \exp \left\lfloor\left(-\mathbf{v}^{2} / \alpha^{2}\right)\left(\left(\mathbf{v}_{\|}-\mathbf{v}_{d}\right)^{2} / \alpha_{\|}^{2}\right)\right\rfloor
\end{aligned}
$$

where $j=$ loss-cone index; $\alpha_{\perp s}$ and $\alpha_{\| s}$ are thermal velocities; for $j=0$ and it reduces to bi-maxwellian and further for $\alpha_{\perp}=\alpha_{\|}$it becomes a Maxwellian. We take $j=0$ bimaxwellian for background plasma and $j=1$ loss-cone for beam plasma. The first order perturbed distribution function $f_{1 s}$ is written as (Misra and Pandey, 1995).

$$
\begin{aligned}
f_{S}(r, \mathbf{v}, t)= & \left(-e_{s} / m_{s} \omega\right) \sum_{m, n, p, q=-\infty}^{\infty} \\
& \cdot\left[\left(J / p\left(\lambda_{2}\right) J_{m}\left(\lambda_{1}\right) J_{p}\left(\lambda_{3}\right) e^{i(\mathbf{k}, \mathbf{r}-\omega t)}\right) /\right. \\
& \left\{\left(\omega-\mathbf{k}_{\|} \mathbf{v}_{\|}-(n+q) \omega_{c s}+p v\right\}\right] \\
& \cdot E_{1 x} J_{n} J_{p}\left\{\left(n / \lambda_{1}\right) U^{*}+\left(p / \lambda_{2}\right) D_{1}\right\} \\
& \left.-i E_{1 y}\left\{J_{n}^{\prime} J_{p} C+J_{n} J_{p}^{\prime} D_{2}\right\}+E_{1 z} J_{n} J_{p} W^{*}\right]
\end{aligned}
$$

where,

$$
C_{1}=\left(1 / \mathbf{v}_{\perp}\right)\left(\delta f_{0} / d \mathbf{v}_{\perp}\right)\left(\omega-\mathbf{k}_{\perp} \cdot \mathbf{v}_{\|}\right)+\left(\delta f_{0} / \delta \mathbf{v}_{\|}\right) \mathbf{k}_{\|}
$$

$$
\begin{aligned}
U^{*}= & C_{1}\left[\mathbf{v}_{\perp}-\left\{v \Gamma_{x} /\left(\omega_{c s}^{2}-v^{2}\right)\right\}\right] \\
W^{*}= & {\left[\left(n \omega_{c s} \mathbf{v}_{\|} / \mathbf{v}_{\perp}\right)\left(\delta f_{0} / \delta \mathbf{v}_{\perp}\right)-n \omega_{c s}\left(\delta f_{0} / \delta \mathbf{v}_{\|}\right)\right] } \\
& \cdot\left[1+\left\{\mathbf{k}_{\perp} \Gamma_{x} v /\left(\omega_{c s}^{2}-v^{2}\right)\right\}\left\{\left(p / \lambda_{2}\right)-\left(n / \lambda_{1}\right)\right\}\right] \\
D_{1}= & C_{1}\left\{v \Gamma_{x} /\left(\omega_{c s}^{2} v^{2}\right)\right\}, \quad J_{n}^{\prime}=\left\{d J_{n}\left(\lambda_{1}\right)\right\} d \lambda_{1} \quad \text { and } \\
J_{p}^{\prime}= & \left\{d J_{p}\left(\lambda_{2}\right)\right\} / d \lambda_{2} \\
\lambda_{1}= & \left(\mathbf{k}_{\perp} \mathbf{v}_{\|}\right) /\left(\omega_{c s}\right), \quad \lambda_{2}=\left(\mathbf{k}_{\perp} \Gamma_{x}\right) /\left(\omega_{c s}^{2}-v^{2}\right) \\
\lambda_{3}= & \left(\mathbf{k}_{\perp} \Gamma_{x} v\right) /\left(\omega_{c s}^{2}-v^{2}\right) .
\end{aligned}
$$

Following Misra and Pandey (1995), the conductivity tensor $\|\sigma\|$ is written as

$$
\|\sigma\|=\Sigma \frac{e_{s}^{2}}{m_{s} \omega}\left[\frac{d^{3} \mathbf{v} J_{q}\left(\lambda_{3}\right)\left\|s_{i j}\right\|}{\omega-n \omega_{c}-q \omega_{c}+p \nu}\right]
$$

where

$$
\begin{aligned}
& \left\|s_{i j}\right\|=\left|\begin{array}{ccc}
J_{n}^{2} J_{p} A\left(\frac{n}{\lambda_{1}}\right) v_{\perp} & i J_{n} B v_{\perp} & J_{n}^{2} J_{p} W^{*}\left(\frac{n}{\lambda_{1}}\right) v_{\perp} \\
J_{n} J_{n}^{\prime} J_{p} A v_{\perp} & J_{n} B v_{\perp} & i J_{n} J_{n}^{\prime} J_{p} W^{*} v_{\perp} \\
J_{n}^{2} J_{p} A v_{\|} & i J_{n} B v_{\|} & J_{n}^{2} J_{p} v_{\|} W^{*}
\end{array}\right| \\
& A=\frac{n}{\lambda_{1}} U^{*}+\frac{p}{\lambda_{2}} D_{1}, \quad B=J_{n}^{\prime} J_{p} C_{1}+J_{n} J_{p}^{\prime} D_{1} .
\end{aligned}
$$

From $J=\|\sigma\| \cdot E_{1}$ and two Maxwell's curl equations for the perturbed quantities, one can get the wave equation as

$$
\left[k^{2}-k \cdot k-\frac{\omega^{2}}{c^{2}} \cdot \epsilon(k, \omega)\right] E_{1}=0
$$

where

$$
\left\|\epsilon(k, \omega)=1-\frac{4 \pi}{i \omega}\right\| \sigma(k, \omega)\|\|
$$

is dielectric tensor. After using the Eq. (6) and (2) Eq. (7) becomes

$\left\|\epsilon_{i j}(k, \omega)\right\|=1+\Sigma \frac{4 \pi e_{s}^{2}}{m_{s} \omega^{2}}\left[\frac{d^{3} \mathbf{v} J_{q}\left(\lambda_{3}\right)\left\|s_{i j}\right\|}{\omega-k v-n \omega_{c}-q \omega_{c}+p v}\right]$.

The generalizied dielectric tensor may be written in complete form as

$$
\begin{array}{ccc}
N^{2} \cos ^{2} \theta_{1}+\epsilon_{11} & \epsilon_{12} & N^{2} \cos \theta_{1} \sin \theta_{1}+\epsilon_{13} \\
\epsilon_{21} & N^{2}+\epsilon_{22} & \epsilon_{23} \\
N^{2} \cos \theta_{1} \sin \theta_{1}+\epsilon_{31} & \epsilon_{32} & N^{2} \sin ^{2} \theta_{1}+\epsilon_{33} \\
=0 . &
\end{array}
$$

If we remove the contribution of a-c field above dielectric tensor is similar to Sazhin (1993). After using the limits $\mathbf{k}_{\perp}=\mathbf{k} \sin \theta_{1} \rightarrow 0$ and $\mathbf{k}_{\|}=\mathbf{k} \cos \theta_{1}$ the generalized dielectric tensor becomes simplified tensor and generalized dispersion relation reduces as

$$
\left|\begin{array}{ccc}
-N^{2}+\epsilon_{11} & \epsilon_{12} & 0 \\
-\epsilon_{21} & -N^{2}+\epsilon_{22} & 0 \\
0 & 0 & \epsilon_{33}
\end{array}\right|=0 .
$$


Above expression is rewritten in more convenient form for whistler waves

$$
-N^{4}-2 \epsilon_{11} N^{2}+\epsilon_{11}^{2}+\epsilon_{12}^{2}=0
$$

for electrostatic waves $\epsilon_{33}=0$.

Neglecting the higher power of $N$ therefore resulting generalized dispersion relation becomes as

$$
\epsilon_{11} \pm \epsilon_{12}=N^{2}
$$

Hence the dispersion relation of whistler wave is obtained from this for $n=1, p=1, q=0$ and putting $J_{p}=1$, $J_{q}=1$.

$$
\begin{aligned}
\frac{\mathbf{k}_{\|}^{2} c^{2}}{\omega^{2}}= & +\Sigma \frac{8 \pi e^{2} n_{0}}{m \omega^{2} \alpha_{\perp}^{2(j+1)} j !} \\
& {\left[X_{1} \frac{\omega}{\mathbf{k}_{\|} \alpha_{\|}} Z(\zeta)+X_{2}(1+\zeta Z(\zeta))\right] }
\end{aligned}
$$

where,

$$
\begin{aligned}
X_{1}= & \frac{\alpha_{\perp s}^{2(j+1)} j !}{2}-\frac{v \Gamma_{x s}}{\omega_{c}^{2}-v^{2}} \frac{\alpha_{\perp s}^{2(j+1)}}{4}\left(j-\frac{1}{2}\right) ! \\
X_{2}= & \frac{\alpha_{\perp s}^{2(j+1)} j !}{2}(j+1)\left(\frac{\alpha_{\perp s}^{2}}{\alpha_{\| s}^{2}}-1\right) \\
& -\frac{v \Gamma_{x s}}{\omega_{c}^{2}-v^{2}} \frac{\alpha_{\perp s}^{2(j+1)}}{4}\left((2 j+1) \frac{\alpha_{\perp s}^{2}}{\alpha_{\| s}^{2}}-1\right) \\
& \cdot\left(j-\frac{1}{2}\right) ! \\
\zeta_{s}= & \frac{\omega-\omega_{c}-\mathbf{k}_{\|} v_{\|}+p v}{\mathbf{k}_{\|} \alpha_{\|}} .
\end{aligned}
$$

After substituting

$$
\frac{\mathbf{k}_{\|}^{2} c^{2}}{\omega^{2}} \gg 1 \quad \omega_{p s}^{2}=\frac{4 \pi e_{s}^{2} n_{0}}{m_{s}} .
$$

Assuming $k$ to be real and using an asymptotic expansion of $Z(\zeta)$ in the limit of large value of as

$$
\begin{aligned}
& Z(\zeta)=i \sqrt{\pi} \exp \left(-\zeta^{2}\right)-\frac{1}{\zeta}\left(1+\frac{1}{2 \zeta^{2}}\right) \\
& \|\zeta\| \gg 1 \operatorname{Im}|\zeta| \ll \operatorname{Re}|\zeta| .
\end{aligned}
$$

The Eq. (15) now reduces to

$$
\begin{aligned}
D(k, \omega)= & \frac{k_{\|}^{2} c^{2}}{\omega_{p s}^{2}}+\left\{\frac{1}{\alpha^{2(j+1)} j !}\right\} \\
& \cdot\left[X_{1} \frac{\omega \mathbf{k}_{\|} v_{d}}{\mathbf{k}_{\|} \alpha_{\|}}\left\{\frac{1}{\zeta} \frac{1}{2 \zeta^{3}}\right\} X_{2} \frac{1}{2 \zeta^{2}}\right. \\
& \left.+\left\{X_{1} \frac{\omega \mathbf{k}_{\|} v_{d}}{\mathbf{k}_{\|} \alpha_{\|}}+X_{2} \zeta\right\}\left\{i \sqrt{\pi} \exp \left(\zeta^{2}\right)\right\}\right]
\end{aligned}
$$

Now introducing the following definitions

$$
\begin{aligned}
& \mathbf{k}_{1}=k \cos \theta_{1}, \quad k=\frac{\mathbf{k}_{\|} \alpha_{\|}}{\omega_{c}}, \\
& X_{3}=\frac{\omega}{\omega_{c}}, \quad X_{4}=-\frac{v}{\omega_{c}}, \quad X_{5}=\frac{\omega-\mathbf{k}_{\|} v_{d}}{\omega_{c}} .
\end{aligned}
$$

The Eq. (18) reduces to simpler numerical dimension less form. The growth rate and real frequency are given in dimension less form as:

$$
\begin{aligned}
\frac{\gamma}{\omega_{c}}= & \frac{\frac{\sqrt{\pi}}{\mathbf{k}_{1}}\left(\frac{X_{2}}{X_{1}}-K_{4}\right) K_{3}^{3} \exp \left(-\left(\frac{K_{3}}{\mathbf{k}_{1}}\right)^{2}\right)}{1+X_{4}+\frac{\mathbf{k}_{1}^{2}\left(1+X_{4}\right)}{2 K_{3}^{2}}-\frac{\mathbf{k}_{1}^{2}}{K_{3}}\left(\frac{X_{2}}{X_{1}}-K_{4}\right)} \\
X_{3}= & \frac{\mathbf{k}_{1}}{\beta}\left[K_{2}\left(1+X_{4}\right)+\frac{X_{2}}{X_{1}} \frac{\beta}{2\left(1+X_{4}+X_{5}\right)}\right] \\
& +X_{5} .
\end{aligned}
$$

Where

$$
\begin{aligned}
& \beta=\frac{K_{B} T_{\|} \mu_{0} n_{0}}{B_{0}^{2}}, \quad K_{2}=\frac{\alpha_{\perp i}^{2(j+1)} j !}{4 X_{1}} \\
& K_{3}=1-X_{3}+X_{4}+X_{5}, \quad K_{4}=\frac{X_{3} X_{4}}{X_{3}} .
\end{aligned}
$$

\section{Result and Discussion}

The growth rate for left hand whistler mode instability have been evaluated for various distribution function of electron density specially for distribution index $j=0$, $j=1$ reducible to bi-maxwellian and loss-cone. Following plasma parameters are assumed for Earth's Magnetosphere at $L=6.6, K_{B} T_{\|}=5 \mathrm{keV}, B_{0}=1 \times 10^{-7} \mathrm{~T}$, $n_{0}=5 \times 10^{6} \mathrm{~m}^{-3}, E_{0}=4 \times 10^{-3} \mathrm{~V} / \mathrm{m}, v=0,4 \mathrm{KHz}$, $8 \mathrm{KHz}, \theta_{1}=0^{0}, 20^{0}, 40^{0}, A_{T}=T_{\perp} / T_{\|}-1=0.25,0.5$, $0.75, v_{d}=0,0.12 \alpha_{\|}, 0.24 \alpha_{\|}$. Plasma parameters at (Wong and Smith, 1994) 1 A.U. $K_{B} T_{\|}=10 \mathrm{eV}, B_{0}=5 \times 10^{-9} \mathrm{~T}$, $n_{0}=6 \times 10^{6} \mathrm{~m}^{-3}, E_{0}=4 \times 10^{-3} \mathrm{~V} / \mathrm{m}, v=0,200 \mathrm{~Hz}$, $400 \mathrm{~Hz}, \theta_{1}=0^{0}, 20^{0}, 40^{0}, A_{T}=T_{\perp} \backslash T_{\|}-1=0,0.25$, $0.5, v_{d}=0,0.4 \alpha_{\|}, 0.8 \alpha_{\|}$in both cases a-c frequencies are chosen such that whistler condition are not violated i.e. less than half gyrofrequency.

In Fig. 1(a) anisotropy variation for bi-maxwellian plasma $j=0$ and loss-cone plasma $j=1$ have been shown in case of magnetosphere at $L=6.6$. In case of bi-Maxwellian plasma anisotropy increases the growth rate but maxima marginally shift towards higher $\overline{\mathbf{k}}$ (frequency value). In the case of $j=1$ while the growth rate is increasing with increase in anisotropy but the maxima is shifting towards lower value of $\overline{\mathbf{k}}$ (frequency). Thus upon the choice of distribution function and amount of anisotropy present various whistler frequencies will be generated. This feature remains similar up to angle $0^{0}$ to $20^{\circ}$, however, the background density and temperature chosen may alter the generated frequency range. Temperature anisotropy remains the prime source of seeding the free energy to the plasma. In case of loss-cone the source of free energy is not only temperature anisotropy but also the increase of $\mathbf{v}_{\perp}$ having greater positive slope.

While Fig. 1(b) shows variation of growth rate with $\overline{\mathbf{k}}$ for distribution index $j=0$ (bi-maxwellian) and $j=1$ (losscone) at 1 A.U. for other fixed plasma parameters as shown in caption. The growth rate is still influenced by temperature anisotropy but the maxima shifts towards lower values of $\overline{\mathbf{k}}$ (frequency) for $j=0$ and towards higher values of $\overline{\mathbf{k}}$ (frequency) for $j=1$ opposite to magnetospheric conditions. In case of 1 A.U. growth rate is still possible at 


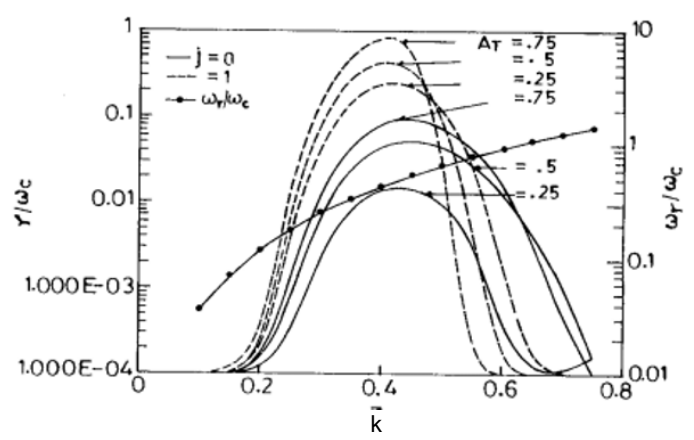

Fig. 1(a). Variation of growth rate $\left(\gamma / \omega_{c}\right)$ with $\overline{\mathbf{k}}$ for different values of temperature anisotropy $A_{T}$ and other fixed plasma parameters $E=4 \times 10^{-3} \mathrm{~V} / \mathrm{m}, v=4 \mathrm{KHz}, \theta_{1}=20^{0}, K_{B} T_{\|}=5 \mathrm{keV}$, $n_{0}=5 \times 10^{6} \mathrm{~m}^{-3}, V_{d} / \alpha_{\|}=0.12, B_{0}=1^{-7} \mathrm{~T}$.

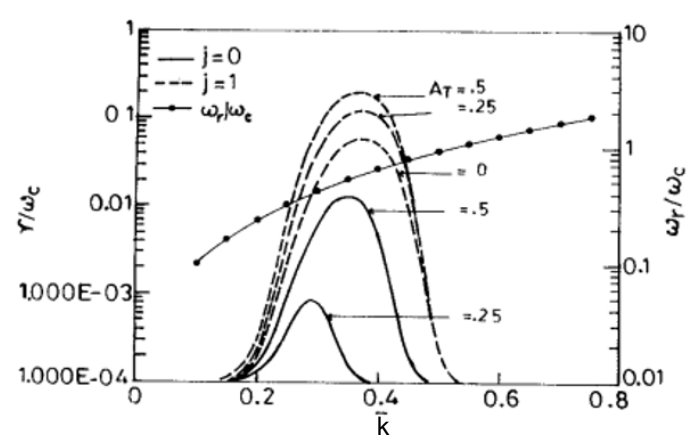

Fig. 1(b). Variation of growth rate $\left(\gamma / \omega_{c}\right)$ with $\overline{\mathbf{k}}$ for different values of temperature anisotropy $A_{T}$ and other fixed plasma parameters $E=4 \times 10^{-3} \mathrm{~V} / \mathrm{m}, v=200 \mathrm{~Hz}, \theta_{1}=30^{0}, K_{B} T_{\|}=10 \mathrm{eV}$, $n_{0}=6 \times 10^{6} \mathrm{~m}^{-3}, V_{d} / \alpha_{\|}=0.8, B_{0}=5 \times 10^{-9} \mathrm{~T}$.

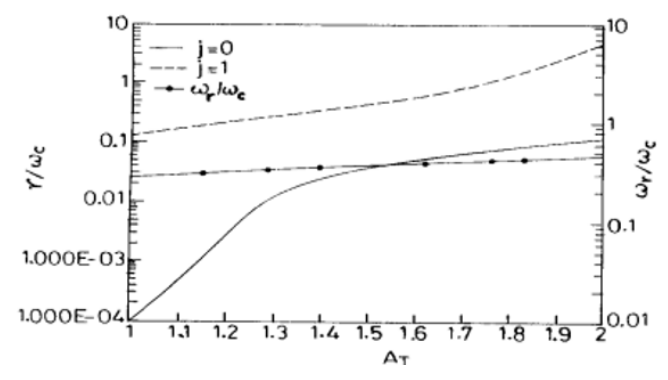

Fig. 2. Variation of growth rate $\left(\gamma / \omega_{c}\right)$ with temperature anisotropy $A_{T}$ and other fixed plasma parameters $E=4 \times 10^{-3} \mathrm{~V} / \mathrm{m}, v=4 \mathrm{KHz}$, $\theta_{1}=20^{0}, K_{B} T_{\|}=5 \mathrm{keV}, n_{0}=5 \times 10^{6} \mathrm{~m}^{-3}, V_{d} / \alpha_{\|}=0.12$, $B_{0}=1 \times 10^{-7} \mathrm{~T}, k=0.4$.

$j=1$ for zero temperature anisotropy because of $\mathbf{v}_{\perp}$ having positive slope and index increasing to $(2 j+1)$ resulting in numerical multiplication to a-c frequency term. Further this situation may also occur due to oblique propagation. Thus different frequencies would be generated at $1 \mathrm{~A}$. U. the frequency of generated whistler waves are $\omega_{r} / \omega_{c}=0.4,0.53$ at $A_{T}=0.25,0.5$ respectively. Wong and Smith (1994) results (their figure no. 6) for generation of whistler waves are in agreement with our results. Further, in case of magnetospheric plasma Fig. 2 describes the maximum growth rate

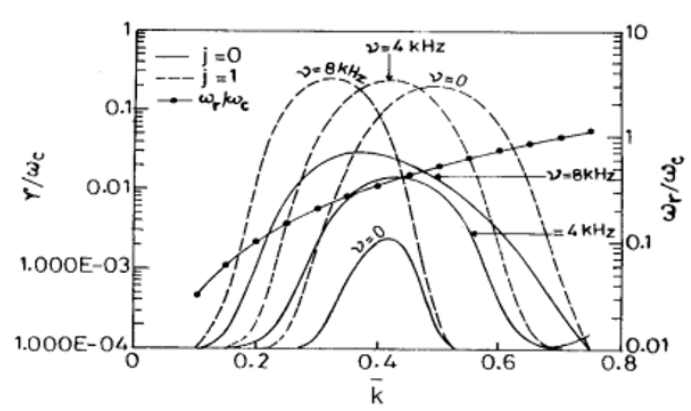

Fig. 3(a). Variation of growth rate $\left(\gamma / \omega_{c}\right)$ with $\overline{\mathbf{k}}$ for different values of a.c. frequency $v$ and other fixed plasma parameters $E=4 \times 10^{-3} \mathrm{~V} / \mathrm{m}$, $A_{T}=0.25, \theta_{1}=20^{0}, K_{B} T_{\|}=5 \mathrm{keV}, n_{0}=5 \times 10^{6} \mathrm{~m}^{-3}, V_{d} / \alpha_{\|}=0.12$, $B_{0}=1 \times 10^{-7} \mathrm{~T}$.

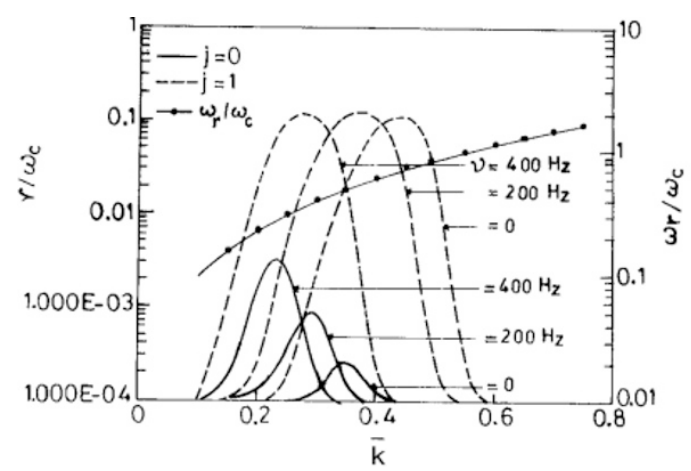

Fig. 3(b). Variation of growth rate $\left(\gamma / \omega_{c}\right)$ with $\overline{\mathbf{k}}$ for different values of a.c. frequency $v$ and other fixed plasma parameters $E=4 \times 10^{-3} \mathrm{~V} / \mathrm{m}$, $A_{T}=0.25, \theta_{1}=30^{0}, K_{B} T_{\|}=10 \mathrm{eV}, n_{0}=6 \times 10^{6} \mathrm{~m}^{-3}, V_{d} / \alpha_{\|}=0.8$, $B_{0}=5 \times 10^{-9} \mathrm{~T}$.

with anisotropy with fixed plasma parameter listed in caption. In case of bi-maxwellian distribution the growth rate first increase and then start saturating for the higher value of temperature anisotropy, whereas for $j=1$ the growth rate is still increasing for much larger temperature anisotropy because of increase in positive slope of $\mathbf{v}_{\perp}$ in addition to temperature anisotropy. Figure 3(a), shows variation of growth rate with $\overline{\mathbf{k}}$ for fixed plasma parameters shown in caption for magnetospheric plasma. The growth rate with a-c frequency increases and maxima shifts towards lower $\overline{\mathbf{k}}$ values in both cases of distributions. Thus covering a wide spectrum of frequencies. The periodic emission consisting of a series of equally spaced discrete emissions were observed at low latitude stations of magnetospheric origin (Dowden, 1962; Lalmani et al., 2000) and at mid latitude by Helliwell (1965). Halliwell (1967) explained these observations based on oblique propagation of whistlers generated at magnetospheric heights. Further, explanations were based on transverse resonance condition and theory based on interaction between energetic streaming electrons with whistler mode waves travelling in opposite directions. Whereas Fig. 3(b) depicts the case at 1 A.U. the width of maxima becomes narrower and shifts towards much lower $\overline{\mathbf{k}}\left(\omega_{r} / \omega_{c}=0.5,0.4\right.$, 0.3 at $v=0,200 \mathrm{~Hz}, 400 \mathrm{~Hz}$ for $j=0$ and $\omega_{r} / \omega_{c}=0.72$, $0.51,0.4$ at $v=0,200 \mathrm{~Hz}, 400 \mathrm{~Hz}$ for $j=1$ respectively) values. The generated frequencies obtained are in agreement 


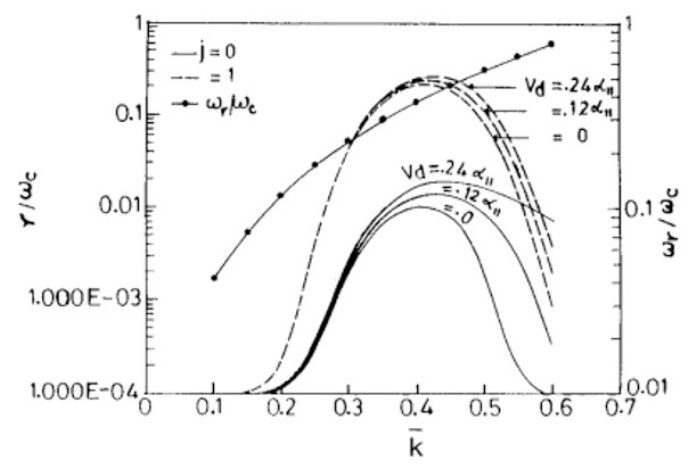

Fig. 4(a). Variation of growth rate $\left(\gamma / \omega_{c}\right)$ with $\overline{\mathbf{k}}$ for different values of electron drift $\left(V_{d}\right)$ and other fixed plasma parameters $E=4 \times 10^{-3} \mathrm{~V} / \mathrm{m}$, $v=4 \mathrm{KHz}, \theta_{1}=20^{0}, K_{B} T_{\|}=5 \mathrm{keV}, n_{0}=5 \times 10^{6} \mathrm{~m}^{-3}, A_{T}=0.25$, $B_{0}=1 \times 10^{-7} \mathrm{~T}$.

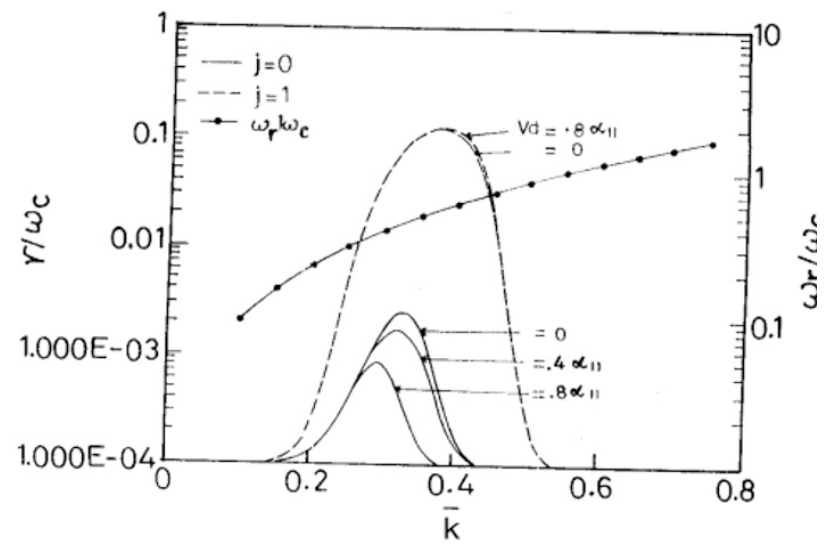

Fig. 4(b). Variation of growth rate $\left(\gamma / \omega_{c}\right)$ with $\overline{\mathbf{k}}$ for different values of electron drift $\left(V_{d}\right)$ and other fixed plasma parameters $E=4 \times 10^{-3} \mathrm{~V} / \mathrm{m}$, $v=200 \mathrm{~Hz}, \theta_{1}=30^{0}, K_{B} T_{\|}=10 \mathrm{eV}, n_{0}=6 \times 10^{6} \mathrm{~m}^{-3}, A_{T}=0.25$, $B_{0}=5 \times 10^{-9} \mathrm{~T}$.

with the results at 1 A.U. obtained by Smith et al. (1991) and Wong and Smith (1994) (Their figure no. 1) in conformity with our calculation. Similar high frequency whistler waves $(10-100 \mathrm{~Hz})$ have been observed by ISEE-3 in the distant up stream plasma (Kennel et al., 1980). It was argued by kennel et al. (1980) that they possibly result from streaming electrons with solar wind in accordance with the instability analysis of Gary and Feldman (1977). In this case also effects of back ground plasma is clearly visible. Figure 4(a) shows variation of growth rate for various values of drift velocity of electrons parallel to the magnetic field in case of magnetospheric plasma. Drift velocity variation increases the growth rate in case of bi-maxwellian plasma and marginally affects growth rate for loss-cone plasma. Figure 4(b) shows the similar variation at 1 A.U. Further growth rate decreases as drift velocity increases for bi-maxwilliam plasma. Drift velocity does not effect growth rate for losscone in this case. Figures 5(a) and 5(b) gives the variation of growth rate for various values of angle for magnetospheric plasma and 1 A.U. interplanetary plasma, respectively. Growth rate from $0^{0}-20^{0}$ is marginally affected and electron remains resonant with left hand polarized mode but

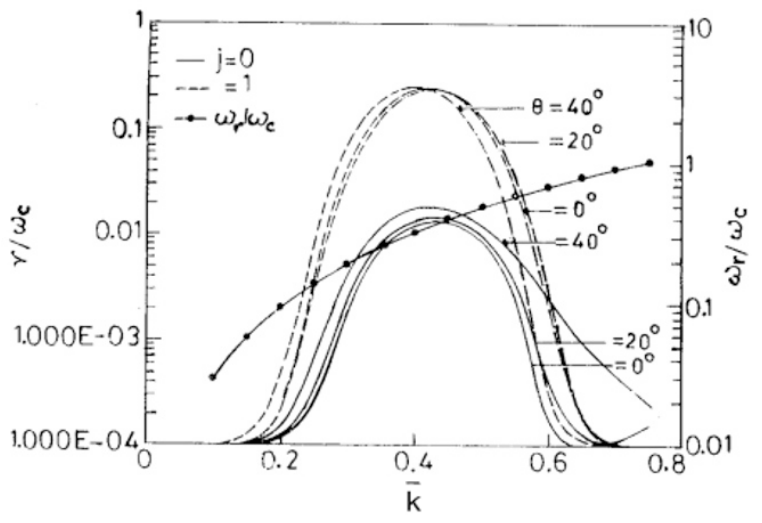

Fig. 5(a). Variation of growth rate $\left(\gamma / \omega_{c}\right)$ with $\overline{\mathbf{k}}$ for different values of angle of propagation $\left(\theta_{1}\right)$ and other fixed plasma parameters $E=4 \times 10^{-3}$ $\mathrm{V} / \mathrm{m}, v=4 \mathrm{KHz}, A_{T}=0.25, K_{B} T_{\|}=5 \mathrm{keV}, n_{0}=5 \times 10^{6} \mathrm{~m}^{-3}$, $V_{d} / \alpha_{\|}=0.12, B_{0}=1 \times 10^{-7} \mathrm{~T}$.

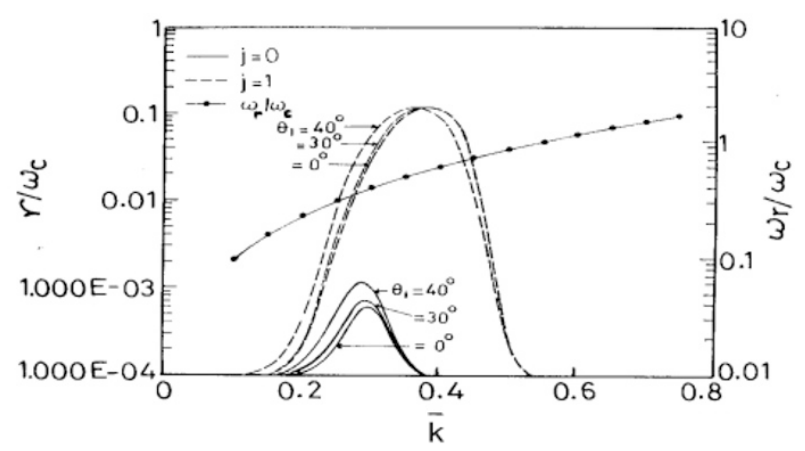

Fig. 5(b). Variation of growth rate $\left(\gamma / \omega_{c}\right)$ with $\overline{\mathbf{k}}$ for different values of angle of propagation $\left(\theta_{1}\right)$ and other fixed plasma parameters $E=4 \times 10^{-3} \mathrm{~V} / \mathrm{m}, v=200 \mathrm{~Hz}, \theta_{1}=30^{0}, A_{T}=0.25, K_{B} T_{\|}=10 \mathrm{eV}$, $n_{0}=6 \times 10^{6} \mathrm{~m}^{-3}, V_{d} / \alpha_{\|}=0.8, B_{0}=5 \times 10^{-9} \mathrm{~T}$.

it increases beyond $20^{\circ}$. After $40^{\circ}$ the electrostatic condition start dominating and non-resonant instability starts contributing or even change in polarization may take place making it a right hand mode. At 1 A.U. low frequency emission of whistler waves for bi-Maxwellian plasma and higher frequency whistler wave for loss-cone is possible. Our results are in agreement with Wong and Smith (1994). However, here we do not get two peaks in absence of the beam.

In order to test the beam effect on simultaneous generation of several whistler waves at 1 A.U. the back ground plasma is chosen with $j=0$ bi-Maxwellian having a-c field and beam as loss-cone $j=1$ without a-c field and results are shown in Fig. 6(a), for fixed plasma parameters shown in caption and for different angles of propagation. Here simultaneous presence of two peaks are seen at 1 A.U. The electron temperature anisotropy remains the main source of instability for left hand instability. However, in the presence of beam component of distribution the modification of usual real frequency results in generation of a new wave. Double peak appears only after 30 when beam temperature anisotropy is 2 or more than 2. Here either electrostatic whistler modes are generated or polarization is getting reversed. When the background plasma is assumed to be 


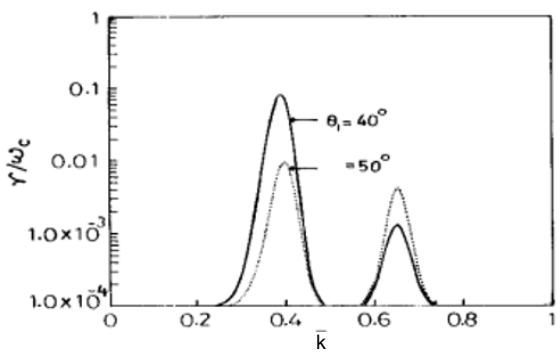

Fig. 6(a). Variation of growth rate $\left(\gamma / \omega_{c}\right)$ with $\overline{\mathbf{k}}$ for different values of angle of propagation $\left(\theta_{1}\right)$ and other fixed plasma parameters. Parameters for background plasma $j=0, E=4 \times 10^{-3} \mathrm{~V} / \mathrm{m}, v=200 \mathrm{~Hz}$, $A_{T}=0.25, K_{B} T_{\|}=10 \mathrm{eV}, n_{0}=6 \times 10^{6} \mathrm{~m}^{-3}, V_{d} / \alpha_{\|}=0.8$, $B_{0}=5 \times 10^{-9} \mathrm{~T}$, and parameters for beam plasma $j=1, K_{B} T_{\|}=1$ $\mathrm{keV}, n_{0}=6 \times 10^{5} \mathrm{~m}^{-3}, V_{d} / \alpha_{\|}=0.8, A_{T}=2$.

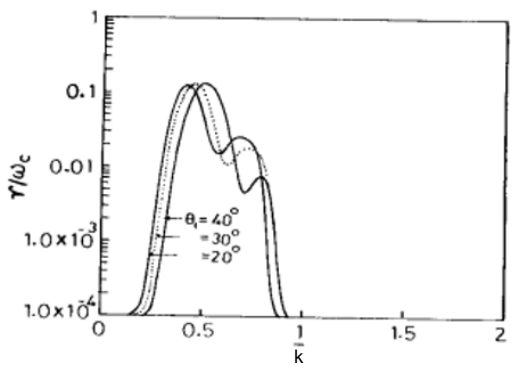

Fig. 6(b). Variation of growth rate $\left(\gamma / \omega_{c}\right)$ with $\overline{\mathbf{k}}$ for different values of angle of propagation $\left(\theta_{1}\right)$ and other fixed plasma parameters. Parameters for background plasma $j=1, E=4 \times 10^{-3} \mathrm{~V} / \mathrm{m}, v=200 \mathrm{~Hz}$, $A_{T}=0.25, K_{B} T_{\|}=10 \mathrm{eV}, n_{0}=6 \times 10^{6} \mathrm{~m}^{-3}, B_{0}=5 \times 10^{-9} \mathrm{~T}$, and parameters for beam plasma $j=0, K_{B} T_{\|}=1 \mathrm{keV}, n_{0}=6 \times 10^{5} \mathrm{~m}^{-3}$, $V_{d} / \alpha_{\|}=0.8, A_{T}=0.25$.

loss-cone type of very low energy and beam is assumed biMaxwelliam of high energy $1 \mathrm{keV}$ or more the double peaks are appearing at all angles less than 50. However, they are not well separated as shown in Fig. 6(b). In earlier case waves are emitted only in limited range of $\overline{\mathbf{k}}$ (frequency) while in second case between the peaks growth reduces but never goes to zero. In this case it remains in left hand polarized mode. The results are in agreement with satellite observations of ISEE-3 and results reported by Smith et al. (1991) and Wong and Smith (1994).

\section{Conclusion}

The effect of distribution functions of back ground plasma influences the generation of oblique whistler waves more effectively at 1 A.U. than at magnetosphere. The a-c frequency alters the range of generated whistler frequencies. At 1 A.U. when background plasma is bi-maxwellian and beam is loss-cone whistler mode generated instability gave two clearly separated peaks. While at 1 A.U. itself when back ground plasma is loss-cone type and beam is biMaxwelliam then two peaks appear with in same unstable spectrum. Thus choice of back ground plasma and beam plays vital role in generating different whistler frequencies although not reported here such separate peaks are obtained in magnetosphere at different angles of propagation only when background plasma has kappa distribution of high energy. The reported results are in conformity with ground based, satellite and reported results.

\section{References}

Block, L. P. and C. G. Falthammer, the role of magnetic field Aligned electric fields in Auroral Acceleration, J. Geophys. Res., 95, 5877-5888, 1990.

Borda de Agua, L., Y. Omura, and H. Matsumoto, Competing processes of plasma wave instabilities driven by an anisotropic electron beam: Linear results and two-dimensional particle simulation, J. Geophys. Res., 101, 15475-15490, 1996.

Devine, P. E., S. C. Chapman, and J. W. Eastwood, One and two dimensional whistler simulations, J. Geophys. Res., 100, 17189-17203, 1995.

Dowden, R. L., Doppler-Shifted cyclotron radiation from electrons, A theory of VLF emissions, J. Geophys. Res., 67, 1745, 1962.

Farrugia, C. J., R. P. Rijnbeck, M. A. Saunders, D. J. Southwood, D. J. Rodgers, M. F. Simth, D. S. Chaloner, D. S. Hall, P. J. Christiansen, and L. J. C. Williscroff, Kulti-instrument study of flux transfer event structure, J. Geophys. Res., 93, 14465-14475, 1988.

Gary, S. P., The mirror and Ion-cyclotron anisotropy instabilities, J. Geophys. Res., 97, 8519, 1992.

Gary, S. P. and W. A. Feldman, Solar wind heat flux regulation by the whistler instability, J. Geophys. Res., 82, 1087-1094, 1997.

Gary, S. P. and C. D. Madland, Electromagnetic electron temperature anisotropy instabilities, J. Geophys. Res., 90, 7607-7610, 1985.

Gary, S. P., S. A. Fuselier, and B. J. Anderson, Ion anisotropy instabilities in the magnetosheath, J. Geophys. Res., 98, 1981, 1993.

Helliwell, R. A., Whistler and related ionospheric phenomena, pp. 255 305, Stanford University Press, Stanford, Calif., 1965.

Helliwell, R. A., A theory of discrete VLF emissions from the magnetosphere, J. Geophys. Res., 72, 4773, 1967.

Kennel, C. F., F. L. Scarf, F. V. Coroniti, R. W. Freder Icks, D. A. Gurnett, and E. J. Smith, Correlated whistler and electron plasma oscillation bursts detected on ISEE3, Geophys. Res. Lett., 7, 129-132, 1980.

Kennel, C. F., F. V. Coroniti, and F. L. Scarf, Plasma waves in magnetotail flux ropes, J. Geophys. Res., 91, 1424-1438, 1986.

Kimura, I. and T. Matsuo, Wave normal direction of auroral hiss observed by the S-310A-5 rocket, Memoirs of the National Institute of Polar Research, Tokyo Special Issue No. 22, pp. 185-195, 1982.

Korth, A., G. Kremser, S. Peraut, and A. Roux, Interaction of particles with Ion-Cyclotron waves and magnetosonic waves, observations from GEOS1 and GEOS2, Planet, Space Sci., 32, 1393, 1984.

LaBelle, J. and R. A. Treumann, Plasma waves at the dayside magnetopause, Space Science Reviews, 47, 175-202, 1988.

Lalmani, M. K. B., R. Kumar, R. Singh, and A. K. Gwal, Expemely small dispersion whistlers and VLF emissions recorded during day time at Jammu, Indian J. Radio 8 Space Phys., 28, 216, 1999.

Lalmani, M. K. B., R. Kumar, R. Singh, and A. K. Gwal, An explanation of day time discrete VLF emissions observed at Jammu $(L=1.17)$ and determination of magnetosphereic parameters, Indian J. Phys., 74 B(2), $117,2000$.

Lindqvist, P. A. and F. S. Mozer, The average tangential electric field at the noon manetopause parameters, J. Geophys. Res., 95, 17137, 1990.

Lyu, L. H. and J. R. Kan, Ion leakage, Ion reflection, Ion heating and shock reformation in a simulated supercritical quasi-parallel collisionless shock, Geophys. Res. Lett., 17, 1041, 1990.

Maynard, N. C., W. J. Burke, and G. R. Wilson, Solar wind control of the penetration of lectric fields in the inner magnetosphere, Adv. Space. Res., 25(7/8), 1393-1396, 2000.

Misra, K. D. and T. Haile, Effect of a-c. electric field on the whistler mode instability in the magnetosphere, J. Geophys. Res., 98, 9297, 1993.

Misra, K. D. and R. S. Pandey, Generation of whistler emissions by injection of hot electrons in the presence of a perpendicular a-c electric field J. Geophys. Res., 100, 19405-19411, 1995.

Misra, K. D. and B. D. Singh, On the modification of the whistler mode instability in the magnetosphere in the presence of a parallel electric field by cold plasma a injection, J. Geophys. Res., 85, 5138, 1980.

Misra, K. D. and B. D. Singh, Electric field induced instability in the magnetosphere, J. Geophys. Res., 82, 2267, 1977.

Misra, K. D., B. D. Singh, and S. P. Mishra, Effects of parallel electric field on whistler mode instability in the magnetosphere, J. Geophys. Res., 84, 5923, 1979 . 
Mozer, F. S., R. B. Torbert, U. V. Fahleson, C. Falthammer, A. Gonfalone, A. Pedssen, and C. T. Russel, Electric field measurements in the solar wind bow shock, magnetosphere, magnetopause and magnetosphere, Space Sci. Rev., 22, 794, 1978.

Pantellini, F. G., E. A. Heron, J. C. Adam, and A. Mangeney, The role of the whistler precursor during the electric reformation of a quasi-parallel shock, J. Geophys. Res., 97, 1303-1311, 1992.

Rycroft, M. J., VLF emissions in magnetosphere, Radio Sci., 7, 811-830, 1972.

Sazhin, S., Oblique whistler mode growth rate and damping in a hot anisotropic plasma, Planet, Space Sci., 36, 1111-1119, 1988.

Sazhin, S., whistler-mode waves in a hot plasma, Cambridge atmosphereic and space science series, Cambridge Univ. Press, New York, 1993.

Shah, H. M., D. S. Hall, and C. P. Chaloner, The electron experiment on the AMPTE UKS, IEEE Trasaction on Geoscience and Remote sensing, GE-23, 292-300, 1985.

Smith, C. W., H. K. Wong, and M. L. Goldstein, Whistler waves associated with the uranian bow shock: out bound observation, J. Geophys. Res., 96, 15841-15852, 1991

Thomas, V. A., D. Winske, and N. Omidi, Reforming supercritical quasiparallel shocks, 1, one and two dimensional simulations, J. Geophys. Res., 95, 18809, 1990.

Tsurutani, B. T., E. J. Smith, R. M. Thorne, R. R. Anderson, D. A. Gurnett, G. K. Parks, C. S. Lin, and C. T. Russel, Wave particle interaction at the magnetopause: Contribution to the dayside aurora, Geophys. Res. Lett., 8, 183-186, 1981.

Tsurutani, B. T., A. L. Brinca, E. J. Smith, R. T. Okida, R. R. Anderson, and T. E. Eastmen, A statistical study of ELF-VLF plasma waves at magnetopause, J. Geophys. Res., 79, 118-127, 1989.

Ward, A. K., D. A. Bryant, T. Edwards, D. J. Parker, A. Ohea, T. J. Patrick, P. H. Sheather, K. P. Barnsdala, and A. M. Cruise, The AMNPTE-UKS space craft, IEEE Transactions on Geoscience and Remote sensing, GE23, 202-211, 1985.

Winske, D., N. Omidi, K. B. Quest, and V. A. Thomas, Reforming supercritical quasi-parallel shocks, 2, Mechanism for wave generation and front reformation, J. Geophys. Res., 95, 18821, 1990.

Wong, H. K. and C. W. Smith, Electron beam excitation of upstream waves in the whistler mode frequency range, J. Geophys. Res., 99, 1337313387, 1994.

Wygant, J. R., M. Bensadoum, and F. S. Mozer, Electric field measurements at sub-critical, oblique bow shock crossings, J. Geophys. Res., 92, 11, 109, 1987.

Zhang, Y. L., H. Matsumoto, and Y. Omura, Linear and non-linear interactions of an electron beam with oblique whistler and electrostatic waves in the magnetosphere, J. Geophys. Res., 98, 21353-21363, 1993.

R. S. Pandey and K. D. Misra (e-mail: kdmisra@banaras.ernet.in) 